STRUCTURAL SCIENCE CRYSTAL ENGINEERING MATERIALS

ISSN 2052-5206

Received 3 March 2020

Accepted 1 July 2020

Edited by P. Macchi, University of Bern, Switzerland

Keywords: $\mathrm{GdVO}_{4}$; first-principles DFT; crystal morphology.

Supporting information: this article has supporting information at journals.iucr.org/b

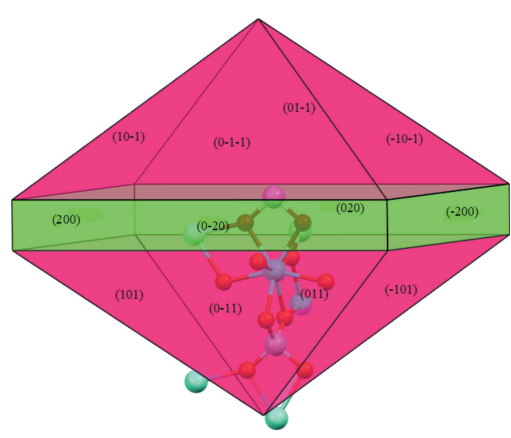

C 2020 International Union of Crystallography

\section{Morphology of the $\mathrm{GdVO}_{4}$ crystal: first-principles studies}

\author{
Emiliana-Laura Andreici Eftimie, ${ }^{a}$ Nicolae M. Avram, ${ }^{\mathrm{a}, \mathrm{b} *} \mathrm{Christian} \mathrm{Jelsch}^{\mathrm{c}}$ and \\ Mirela Nicolov ${ }^{\mathrm{d}}$
}

\begin{abstract}
${ }^{\mathbf{a}}$ Faculty of Physics, West University of Timisoara, V. Parvan 4, Timisoara, 300223, Romania, ${ }^{\mathbf{b}}$ Academy of Romanian Scientists, Independentei Street 54, Bucharest, 050094, Romania, ' CNRS, CRM2, Université de Lorraine, Nancy,

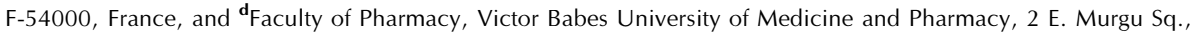
Timisoara, 300041, Romania. *Correspondence e-mail: nicolae.avram@e-uvt.ro
\end{abstract}

The present paper reports a theoretical investigation based on first-principles density functional theory calculations to predict the external morphology of the tetragonal $\mathrm{GdVO}_{4}$ crystal from its internal structure. The Bravais-FriedelDonnay-Harker (BFDH) method, attachment energy (AE) method and surface energy (SE) method were used in this study. Slice energies (cohesive, attachment and specific surface) of the three main crystal faces having (110), (101) and (200) orientation and their $d_{h k l}$ thicknesses were computed using CRYSTAL17 code, in the frame of a $2 \mathrm{D}$ periodic slab model. The relative growth rate $\left(R_{h k l}\right)$ and the morphological importance $\left(\mathrm{MI}_{h k l}\right)$ for each unrelaxed and relaxed $(h k l)$ face of interest were determined. Consequently, the crystal shapes predicted based upon BFDH, AE and SE methods were represented by the Wulff construction. The results of the morphology crystal predictions, based on the above methods, were compared both against each other and against the experimentally observed morphologies. A quite satisfactory agreement between the predicted and observed crystal morphologies is noticed.

\section{Introduction}

Gadolinium orthovanadate $\left(\mathrm{GdVO}_{4}\right)$ belongs to the group of rare-earth orthovanadates $\left(\mathrm{REVO}_{4}\right)$, where $\mathrm{RE}$ are rare-earth elements, including lanthanoids from $\mathrm{La}$ to $\mathrm{Lu}$ and also $\mathrm{Y}$ and Sc. $\mathrm{GdVO}_{4}$ has attracted worldwide attention as a multifunctional material due to the half-filled $4 f$ shell of gadolinium, which plays an important role in the field of inorganic compounds. As a tetragonal crystal system, having $14_{1} /$ amd space group and zircon-type structure, $\mathrm{GdVO}_{4}$ exhibits chemical stability, high thermal conductivity, luminescent and magnetic properties (Szczeszak et al., 2014). This material can be doped with trivalent lanthanide ions $\left(\mathrm{Dy}^{3+}, \mathrm{Eu}^{3+}, \mathrm{Er}^{3+}\right.$, $\left.\mathrm{Nd}^{3+}, \mathrm{Yb}^{3+}, \mathrm{Tm}^{3+}, \mathrm{Ho}^{3+}, \mathrm{Sm}^{3+} \ldots\right)$, due to the similar ionic radii between $\mathrm{Gd}^{3+}$ and other $\mathrm{RE}\left(\mathrm{RE}^{3+}\right)$ cations. Therefore, $\mathrm{GdVO}_{4}$ could be used as a laser, phosphor or up-convertor (Cho, 2013; Yu et al., 2013; Yan \& Gu, 2009; Shanta Singh et al., 2012; Tang et al., 2012; Cante et al., 2018; Shimamura et al., 1996; Rabasovic et al., 2018; Qiao et al., 2017; Min \& Jung, 2019; Jovanović et al., 2018; Zheng et al., 2011; Gavrilović et al., 2016). The $\mathrm{Bi}^{3+}$-doped $\mathrm{GdVO}_{4}$ crystal is also of great importance in modern electronics because of unique luminescent characteristics that can be applied in white light emitting diodes (Vasylechko et al., 2018; Krasnikov et al., 2019). In addition, recent efforts show important implications of $\mathrm{GdVO}_{4}$ for photocatalytic reactions since it possesses a strong ability to generate hydrogen from water or water/alcohol 
solutions (Oshikiri et al., 2014a,b; Mazierski et al., 2019). Many studies have demonstrated that downscaled systems of $\mathrm{GdVO}_{4}$ exhibit multiple characteristics in the field of biomedical applications: photodynamic therapy, radiation therapy, drug delivery etc. (Teo et al., 2016; Belkina et al., 2016; Dong et al., 2015). There are many preparation methods that have been reported in the literature for obtaining $\mathrm{GdVO}_{4}$ such us sol-gel (Chumha et al., 2014), edge-defined film-fed (EFG) (Epelbaum et al., 1998), Czochralski (Loiko et al., 2013), co-precipitation (Vosoughifar, 2017), hydrothermal (Szczeszak et al., 2014; Mahapatra \& Ramanan, 2005), solvothermal (Liang et al., 2011). Among all the processes mentioned above, hydrothermal growth is one of the most promising and widely employed methods, due to the possibility of producing large crystals and the advantage of being able to control the morphology and crystal size.

Various theoretical DFT (density functional theory) studies of $\mathrm{GdVO}_{4}$ have been published regarding electronic structure, Fermi surface, optical properties as well as the elastic properties (Huang et al., 2012; Reshak \& Azam, 2013). Therefore, due to its excellent properties stated above, knowledge of the shape, size and crystal faces is critically important to many industrial processes. In order to obtain high-quality crystals by means of morphology predictions, experimental researchers most often need to visualize a crystal shape resulting from theoretical studies or from a set of experimental data, e.g. specific surface energies or growth rates. Many researchers, over the years, have reported in a series of theoretical and experimental papers the growth form and equilibrium morphology of some ionic solids and molecular crystals (Liu et al., 2019; Zhu et al., 2004; Wang et al., 2010; Zhang, 2014; Singh et al., 2012; Heo et al., 2018; Barbosa et al., 2017; Ribeiro et al., 2019; Stirner et al., 2018; Arrouvela \& Eon, 2019; Goel et al., 2019; Credendino et al., 2009; Bittarello et al., 2018; Nakayama et al., 2013; Oliveira et al., 2016). In all these works, a variety of methods have been employed for the morphology prediction. Here, we only mention those that are of interest to us: Bravais-Friedel-Donnay-Harker (BFDH) rules (Bravais, 1866; Friedel, 1907; Donnay \& Harker, 1937), attachment energy (AE) method (Hartman \& Perdok, 1955; Hartman, 1973; Bennema, 1993; Hartman \& Bennema, 1980; Woensdregt, 1993), surface energy (SE) method and the Wulff construction method (Dovesi et al., 2005; Wulff, 1901). It should be emphasized that all these theoretical methods, which are in continuous development versus a trial-and-error experimental approach, give, in advance, reliable morphology predictions for crystals. However, in spite of the fact that inorganic crystal growth has been intensively investigated, the theoretical predictions of morphology for these crystals are scarce (Dandekar et al., 2013). So, our goal is to bring more insight into the field of theoretical morphology of inorganic crystals, based on the quantum-mechanical computer simulations and modern first-principles methods like DFT. There is a lot of literature on the crystal growth of $\mathrm{GdVO}_{4}$, but there is no prediction using theoretical simulation for the growth and equilibrium form of this crystal. The clue, in these simulations, is related to the specific surface energies $\left(E_{\text {surf }}^{h k l}\right)$, cohesive energies $\left(E_{\text {coh }}\right)$ and attachment energies $\left(E_{\text {att }}^{h k l}\right)$. In the literature, there is one single result that deals with orthovanadate morphology predictions by a semi-empirical force-field method, based on the AE method of Hartman-Perdok theory (Hartman \& Perdok, 1955), for monoclinic $\mathrm{LaVO}_{4}$ and tetragonal $\mathrm{YVO}_{4}$ single crystals (Cong et al., 2010). Furthermore, two articles deal with $E_{\text {surf }}^{\text {hkl }}$ calculations and equilibrium shape related to the tetragonal phase of $\mathrm{LaVO}_{4}$ by means of a DFT method (Li et al., 2012; Gouveia et al., 2016).

The aim of this paper is the first-principles calculation of the crystal growth morphology and equilibrium morphology of the $\mathrm{GdVO}_{4}$ crystal in vacuum, based on DFT methodology and the above-mentioned methods. A detailed description of first-principles calculation of $E_{\mathrm{surf}}^{h k l}, E_{\mathrm{coh}}$ and $E_{\mathrm{att}}^{h k l}$ energies is presented and illustrated in this paper. The predicted morphologies from each method are discussed and compared both against each other and against the experimentally observed morphologies.

\section{Theoretical methods}

\subsection{BFDH method}

Bravais in 1866, Friedel in 1907, Dannay and Harker in 1937 founded the BFDH method (Bravais, 1866; Friedel, 1907; Donnay \& Harker, 1937), based merely on the lattice parameters and the symmetry of the crystal. The BFDH method is based on two rules: the first allows us to generate a list of possible crystal growth faces (Donnay \& Harker, 1937) and the second one shows how the growth rate $R_{h k l}$ of a given $(h k l)$ face is calculated (Bravais, 1866; Friedel, 1907). The $R_{h k l}$ of a given face is proportional to the $D_{h k l}$ distance from the center of the crystal to the $(h k l)$ face, in the normal direction (Wulff, 1901) and inversely proportional to $d_{h k l}$ interplanar spacing. An initial approach for $R_{h k l}$ was as given by equation (1) (Friedel, 1907), where $C$ is a rate constant:

$$
R_{h k l}=1 /\left(C d_{h k l}\right) \text {. }
$$

Later, two new equations [equations (2), (3)] were formulated for growth rates (Donnay \& Harker, 1937):

$$
\begin{gathered}
R_{h k l}=\exp \left(-C d_{h k l}\right) \\
R_{h k l}=d_{h k l} \exp \left(-C d_{h k l}\right) .
\end{gathered}
$$

Besides the growth rate $R_{h k l}$, another parameter to characterize a crystal face is the morphological importance $\left(\mathrm{MI}_{h k l}\right)$ of a face. According to Friedel (1911), the $\mathrm{MI}_{h k l}$ of a crystal face is understood as its relative size in a given crystal shape. In the frame of the BFDH model, relation (4) shows that faces with the smaller $D_{h k l}$ [distance from the center of the crystal to $\left(d_{h k l}\right)$ face in the normal direction] have the lowest growth rate and most morphological importance:

$$
R_{h k l} \sim D_{h k l} \sim\left(\mathrm{MI}_{h k l}\right)^{-1} .
$$

In the BFDH approach, no consideration is given when predicting relative growth rates of faces to the atoms, to partial charges, to the bond types or to the interatomic forces. However, this method remains the simplest way to quickly 
identify the possible crystal growth faces and to estimate, at first, the shape of a given crystal.

\subsection{Attachment energy (AE) method}

In the literature, in the field of growth theory, two contributions can be found for predicting the ideal crystal morphology:

(i) Periodic bond chain (PBC) theory of Hartman and Perdok (Hartman \& Perdok, 1955), a method that reflects the morphological importance of various crystal faces.

(ii) Attachment energy (AE) method (Hartman \& Bennema, 1980), a method that provides the relative growth rates of faces $\left(R_{h k l}\right)$.

The attachment energy $E_{\text {att }}^{h k l}$, an important parameter used to understand the nature of chemical bonding in solids, is defined as the energy released upon the addition of a building unit (growth slice) to the growing crystal surface. Within this method, it is assumed that the growth rate $R_{h k l}$ of a face $(h k l)$ is proportional to the attachment energy $E_{\mathrm{att}}^{h k l}$ (Hartman \& Bennema, 1980; Berkovitch-Yellin, 1985; Docherty et al., 1991). The AE method predicts also that $\mathrm{MI}_{h k l}$ is inversely proportional to the $E_{\text {att }}^{h k l}$ of a face. Therefore, this method assumes that faces with the lowest $E_{\text {att }}^{h k l}$ will have the largest $\mathrm{MI}_{h k l}$ and are the slowest growing:

$$
\begin{gathered}
R_{h k l} \sim\left|E_{\mathrm{att}}^{h k l}\right| \\
\mathrm{MI}_{h k l} \sim \frac{1}{\left|E_{\mathrm{att}}^{h k l}\right|} .
\end{gathered}
$$

Unfortunately, $E_{\text {att }}^{h k l}$ is not a directly measurable parameter; however, it is related to a measurable value by the determination of the cohesive energy $E_{\text {coh }}$ for the bulk and slices. In turn, relative to any crystal face, $E_{\mathrm{att}}^{h k l}$ is calculated as follows (Hartman \& Bennema, 1980; Berkovitch-Yellin, 1985; Docherty et al., 1991):

$$
E_{\mathrm{att}}^{h k l}=E_{\mathrm{latt}}-E_{\mathrm{slice}}^{h k l}
$$

where $E_{\text {latt }}$ is the lattice energy of the crystal (often referred to as the binding or cohesive energy of the crystal) and $E_{\text {slice }}^{h k l}$ is the slice energy, i.e. energy released per molecule on the formation of a new growth layer with a thickness of $d_{h k l}$ (Hartman, 1973; Hartman \& Bennema, 1980). The AE method is widely applied to organic and inorganic crystals and can successfully predict the crystal growth morphology, as it takes into account the energetic interactions of the system (Kevin et al., 2018; Massaro et al., 2011; Aquilano et al., 2016).

\subsection{Surface energy (SE) method}

The SE method, known also as the equilibrium morphology method and very common in the field of inorganic compounds, especially when one is interested in the chemical processes occurring on a crystal surface, requires the knowledge of $E_{\text {surf }}^{h k l}$ and the $R_{h k l}$, for all relevant crystal faces, at $0 \mathrm{~K}$. The equilibrium crystal shape can be determined based on Wulff's theorem (Wulff, 1901). Under these conditions, the $R_{h k l}, \mathrm{MI}_{h k l}$ and $E_{\text {surf }}^{h k l}$ values are connected as follows:

$$
\begin{gathered}
R_{h k l} \sim E_{\mathrm{surf}}^{h k l} \\
\mathrm{MI}_{h k l} \sim\left(E_{\mathrm{surf}}^{h k l}\right)^{-1} .
\end{gathered}
$$

The formula to calculate the surface energy is given in equation (10):

$$
E_{\text {surf }}^{h k l}=\left[E(n)_{\text {slab }}^{h k l}-N \times E_{\text {bulk }}\right] / 2 \times A .
$$

Thus, the surface energy defined in equation (10) is the difference between two energy quantities, where $E(n)_{\text {slab }}^{h k l}$ is the energy of a slab with $n$ layers. $N$ represents the number of atoms in the surface slab, $E_{\text {bulk }}$ is the bulk energy per atom and $A$ is the surface area of the slab. Despite its importance, $E_{\mathrm{surf}}^{h k l}$ is difficult to obtain experimentally and cannot be directly measured. Surfaces are sensitive to many factors such as temperature, pressure and dielectric constant, for example. Hence, it is not surprising that experimental measurement of equilibrium shape for a wide range of materials has not yet been performed, probably because the true equilibrium is not reached.

\section{Computational methodology}

All calculations in this article were performed with the $a b$ initio CRYSTAL17 code (Dovesi et al., 2018). This software adopts atom-centered Gaussian-type functions as basis sets to represent crystalline orbitals and implements the HartreeFock and Kohn-Sham self-consistent field (SCF) methods for the treatment of periodic systems. Here, we focus on the assessment of current DFT methods explored on 3D and 2D geometrical structures of $\mathrm{GdVO}_{4}$ to capture the effect of stability that arises from full relaxation of atomic positions and lattice constants.

\subsection{Geometry optimization of the bulk (3D periodicity) and} $E_{\text {latt }}$ computed

$\mathrm{GdVO}_{4}$ is a tetragonal crystal system with zircon-type structure having space group I4 1 /amd (No. 141 in the International Tables of Crystallography) and four chemical formulae per unit cell $(Z=4)$. The unit cell of $\mathrm{GdVO}_{4}$ is presented in Fig. S1 of the supporting information (SI), beside its autostereogram.

Firstly, we have carried out full geometry optimization in order to obtain the ground-state energy of the crystal. The performance of different functionals like SOGGAXC, B3LYP, B3PW, WC1LYP and B3LYP-D3 has been successfully tested. Further computational details and data (Table S1) for lattice parameters, volumes and distances, after the full optimization process, are collected and presented in the SI. The calculations show that, among all of these probed functionals, B3LYP (Becke, 1988; Becke, 1993; Lee et al., 1988) emerged with an average absolute deviation of $0.6 \%$ on the unit-cell volume. Also, the Becke hybrid exchange correlation functional gives reasonable agreement between the calculated energy band gap $4.54 \mathrm{eV}$ (this paper) and the experimental value $3.6 \mathrm{eV}$ (Jovanović et al., 2018). All calculations were carried out taking into account a polarization TZVP basis set (Vilela 
Table 1

The two most important $(h k l)$ faces of the $\mathrm{GdVO}_{4}$ crystal, interplanar distances, growth rates and the morphological importance.

\begin{tabular}{lllr}
\hline Faces $(h k l)$ [multiplicity] & $d_{h k l}(\AA)$ & $R_{h k l} \dagger$ & $\mathrm{MI}_{h k l}$ \\
\hline$(101)[4]$ & 4.7860 & 0.06 & 17.66 \\
$(200)[8]$ & 3.6227 & 0.11 & 8.79 \\
\hline
\end{tabular}

$\dagger$ Calculated with equation (2) and $C=0.6$.

Oliveira et al., 2019) for $\mathrm{V}^{5+}$ and $\mathrm{O}^{2-}$ ions ( $\operatorname{spin} S=0$ ) and a quasi-relativistic ECP28MWB pseudopotential (Desmarais et al., 2018) for $\mathrm{Gd}^{3+}$ ions (in the high-spin state with $S=7 / 2$ ).

Because the morphology predictions are sensitive to the force field, it is necessary to verify if the calculated lattice energy value is relatively close to the experimental one and overall to confirm that the energetics of the crystal are captured. $E_{\text {latt }}$ of a crystal can be defined as (Gavezzotti \& Filippini, 1997)

$$
E_{\text {latt }}=E_{\text {bulk }}-\sum_{\mathrm{i}}^{N} E_{\mathrm{i}}
$$

where $E_{\text {bulk }}$ is the energy of the bulk crystal unit cell, $E_{\mathrm{i}}$ is the energy for each ion belonging to the crystal unit cell and $N$ is the number of ions in the unit cell. From equation (11), one obtains $E_{\text {latt }}=-26816.7259 \mathrm{~kJ} \mathrm{~mol}^{-1}$ per unit formula and the remaining terms are given in Table S2 in the SI. This value cannot be compared with the experimental value of $E_{\text {latt }}$, because it cannot be empirically measured. That is why it is compared using its relation with the lattice enthalpy $\Delta H_{\text {latt }}$ (Jenkins, 2005),

$$
\Delta H_{\text {latt }}=E_{\text {latt }}+2 R T
$$

where $T$ is the temperature at which the lattice enthalpy is measured and $R$ is the gas constant (the magnitude of $R T$ is approximately $2.4789 \mathrm{~kJ} \mathrm{~mol}^{-1}$; at $\left.T=0 \mathrm{~K}, \Delta H_{\text {latt }}=E_{\text {latt }}\right)$. It is important to note that, in equation (12), both quantities $\Delta H_{\text {latt }}$ and $E_{\text {latt }}$ are related by a correction term $2 R T$, relatively small in magnitude.

According to the Born-Haber thermochemical cycle (Born, 1919; Haber, 1919),

$$
\mathrm{Gd}^{3+}(\mathrm{g})+\mathrm{V}^{5+}(\mathrm{g})+4 \mathrm{O}^{2-}(\mathrm{g}) \rightarrow \mathrm{GdVO}_{4}(\mathrm{~s})
$$

the lattice enthalpy of $\mathrm{GdVO}_{4}$, at $298.15 \mathrm{~K}$ and 760 torr $(1$ torr $=133.322 \mathrm{~Pa})$ pressure, is $26080 \pm 23\left(\mathrm{~kJ} \mathrm{~mol}^{-1}\right)$ (Petrov, 2013). From equation (12), the corresponding value of $E_{\text {latt }}$ is $26075.0422 \pm 23 \mathrm{~kJ} \mathrm{~mol}^{-1}$. The sign of the $\Delta H_{\text {latt }}$ must be reversed if the process takes place in the opposite direction. Frequently $\Delta H_{\text {latt }}$ and $E_{\text {latt }}$ are assumed to be synonymous (Jenkins, 2005). So, one can deduce that the lattice energy from DFT calculation agrees well with the corresponding value deduced from equation (12), as the deviation is only $2.8 \%$.
3.2. Geometry optimization of slabs (2D periodicity) and $\boldsymbol{E}_{\text {slice }}^{h k l}$ computed

To start, an analysis of the forms and planes of the $\mathrm{GdVO}_{4}$ crystal was performed by means of the CRYSTAL17 code (Dovesi et al., 2018) and the results are summarized in Table $\mathrm{S} 3$ in the SI.

In the second step, the main objective was to construct the surfaces corresponding to forms from Table S3, starting from the new optimized geometry of the bulk. Naturally, we employed the procedure implemented in the CRYSTAL17 code (Dovesi et al., 2018) to build bidimensional slabs and to carry out again a new optimization of these slabs. For the slabs relaxation process, we have maintained the computational parameters as we discussed in Section 3.1, barring the shrinking factor along the reciprocal-lattice vectors, here set to 8,8 and $34 k$-points in the first Brillouin zone. The thickness of the $2 \mathrm{D}$ slab is associated with the number of atomic layers. Therefore, slab models containing six, ten and three atomic layers for the (110), (101) and (200) surfaces, respectively, were constructed. Special attention must be paid to the surface termination, because often more than one surface termination is possible. In that case, a slab must have a minimal total energy and surface energy. Representations of the main slabs before relaxation are shown in Figs. S2, S3 and S4 in the SI, drawn with $J$-ice (Canepa et al., 2011), and the slab parameters before and after relaxation are compiled in Table S4 in the SI.

Based on the 2D periodic slab model, a DFT calculation has been performed to compute $E_{\text {slice }}^{h k l}$, i.e. the cohesion energy $E_{\text {coh }}$ per mole of any slab $h k l$ for the most stable faces of the $\mathrm{GdVO}_{4}$ crystal, in accordance with Table S3. All computed results for $E_{\text {slice }}^{h k l}$, regarding the (110), (101) and (200) faces, are listed in Table S5 in the SI.

\section{Results and discussion}

In this section, the theoretical morphology of $\mathrm{GdVO}_{4}$ crystals associated with several low-index faces is explored by means of BFDH, AE and SE methods.

\subsection{Morphology prediction by the BFDH method}

In spite of the fact that CRYSTAL17 ab initio code analysis (Dovesi et al., 2018) shows that the first three most important faces are (110), (101) and (200), the BFDH method, implemented in Mercury software (Macrae et al., 2008), reports only two major faces (101) and (200) which dominate the crystal habit. The (110) face is missing, because the BFDH method explains the morphology based on $d_{h k l}$ values as reproduced in an X-ray diffraction pattern. Therefore, based on this consideration, only two faces are displayed in Table 1 together with $d_{h k l}, R_{h k l}$ and $\mathrm{MI}_{h k l}$.

According to BFDH rules, the larger the interplanar distance $d_{h k l}$ is, the larger the morphological importance of the corresponding $(h k l)$ face is. Thus, the BFDH theory predicts the (101) face to be of highest $\mathrm{MI}_{h k l}$ : $\mathrm{MI}_{101}>\mathrm{MI}_{200}$. The Donnay \& Harker (1937) analysis suggests that the $\mathrm{GdVO}_{4}$ crystal shape is built only from experimental observed forms. 
Table 2

The calculated unrelaxed and relaxed $E_{\text {att }}^{h k l}$ per unit formula, $R_{h k l}$ and $\mathrm{MI}_{h k l}$ of the different shape faces of the $\mathrm{GdVO}_{4}$ crystal in the AE method.

$R_{h k l}$ is $R_{(h k l)} / R_{(110)}$.

\begin{tabular}{llllllll}
\hline $\begin{array}{l}\text { Faces } \\
(h k l)\end{array}$ & $\begin{array}{l}d_{h k l} \\
(\AA)\end{array}$ & $\begin{array}{l}\text { Unrelaxed } \\
E_{\text {att }}^{h k l} \\
\left(\mathrm{~kJ} \mathrm{~mol}^{-1}\right)\end{array}$ & $R_{h k l}$ & $\mathrm{MI}_{h k l}$ & $\begin{array}{l}E_{\text {att }}^{h k l} \\
\left(\mathrm{~kJ} \mathrm{~mol}^{-1}\right)\end{array}$ & $R_{h k l}$ & $\mathrm{MI}_{h k l}$ \\
\hline$(110)$ & 5.1233 & -54.9661 & 1.00 & 1.79 & -37.7750 & 1.00 & 1.25 \\
$(101)$ & 4.7860 & -98.2757 & 1.79 & 1.00 & -39.1714 & 1.04 & 1.20 \\
$(200)$ & 3.6227 & -57.5644 & 1.05 & 1.71 & -47.0335 & 1.25 & 1.00 \\
\hline
\end{tabular}

The two forms are $\{101\}$, consisting of eight faces (101), (101), (011), $(01 \overline{1}),(0 \overline{1} 1),(0 \overline{1}),(\overline{101}),(\overline{10} \overline{1})$, and $\{200\}$ consisting of four faces $(200),(020),(0 \overline{2} 0),(\overline{2} 00)$.

Crystal morphology predicted by this simple model (Fig. 1), strictly based on geometry and symmetry, shows the ideal shape of the $\mathrm{GdVO}_{4}$ crystal as a combination of a short tetragonal prism with a square bipyramid. It was compared with the shape of the $\mathrm{GdVO}_{4}$ synthesized crystals, presented in Fig. 2 (Yan et al., 2013), and was found to be in favorable agreement.

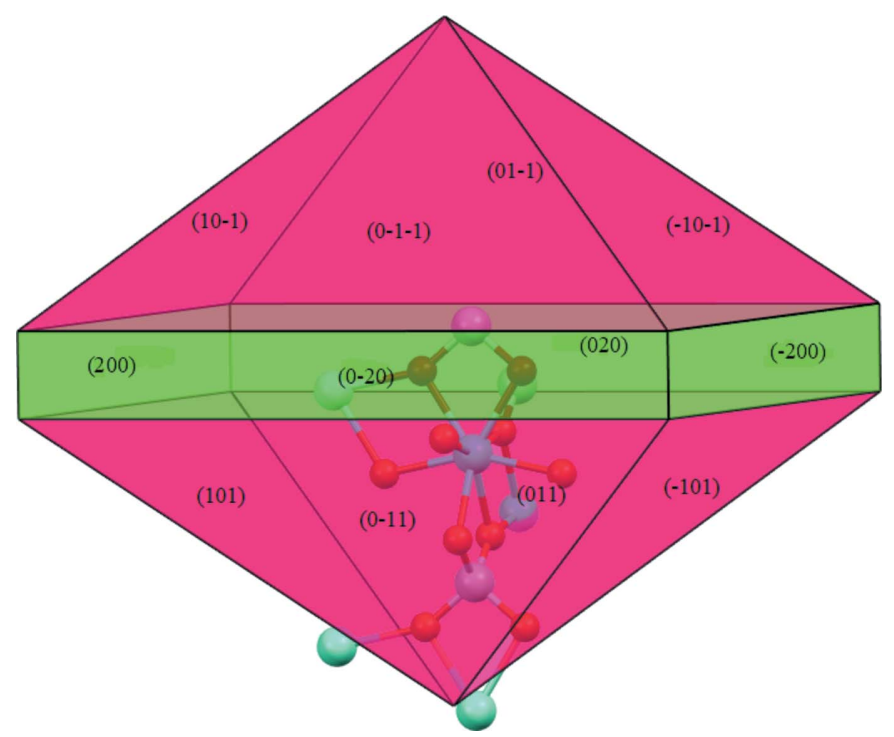

Figure 1

The indexed shape of $\mathrm{GdVO}_{4}$ predicted by BFDH rules, drawn with Mercury software (Macrae et al., 2008).

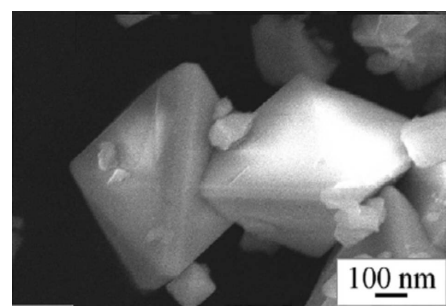

Figure 2

SEM (scanning electron microscopy) micrograph of $\mathrm{GdVO}_{4}: \mathrm{Eu}^{3+}$ crystals synthesized hydrothermally. [Reproduced with the permission of Elsevier from Yan et al. (2013).]
Like in the case of the $\mathrm{GdVO}_{4}$ crystal, the BFDH method shows good agreement with experiment for many crystals (Docherty et al., 1991; Kevin et al., 2018) and can be used for initial prediction of the crystal shapes, before more sophisticated energy calculations. However, the BFDH approach may not always be accurate in predicting the crystal shape. This depends, especially for molecular crystals, on the bonding effect in the crystal. The stronger the bonding effect, the less accurate the method becomes. But in all cases the method is useful to identify the most morphologically important faces needed for shape building.

\subsection{Growth morphology prediction by the AE method}

The calculation of the parameters $E_{\mathrm{att}}^{h k l}, R_{h k l}$ and $\mathrm{MI}_{h k l}$ of the different faces of the $\mathrm{GdVO}_{4}$ crystal, based on equations (6)(8), both for unrelaxed and relaxed faces (110), (101) and (200), was performed and the results are collected in Table 2 .

As can be seen from Table 2, the $E_{\text {att }}^{h k l}$ values decrease upon relaxation and induce a change in $\mathrm{MI}_{h k l}$ arrangement. Clearly, the crystal growth morphology calculated by the AE method predicts the $\mathrm{MI}_{h k l}$ pattern for the shape faces as follows:

(a) Unrelaxed structure: $\mathrm{MI}_{110}>\mathrm{MI}_{200}>\mathrm{MI}_{101}$.

(b) Relaxed structure: $\mathrm{MI}_{110}>\mathrm{MI}_{101}>\mathrm{MI}_{200}$.

On the basis of these results, it appears that the most stable face is (110) for both relaxed and unrelaxed structures, but the relative sequences found for the second and third most stable surfaces (200) and (101) are different in the two predictions.

A morphological sketch of the $\mathrm{GdVO}_{4}$ crystal deduced from the AE model in vacuum can be seen in Fig. 3(a) for the unrelaxed case and Fig. 3(b) for the relaxed case.

From the AE theoretical growth morphology shown in Fig. 3, two different shapes with the same form can be seen: (a) a long bipyramid prism and $(b)$ a short bipyramid prism. The unrelaxed $\mathrm{AE}$ theoretical predictions from Fig. 3(a) show good agreement with the experimental shape in Fig. 4(a) (Min

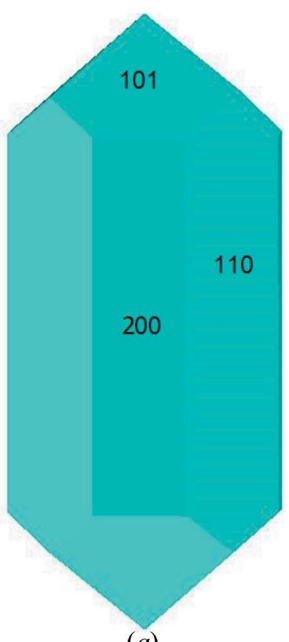

(a)

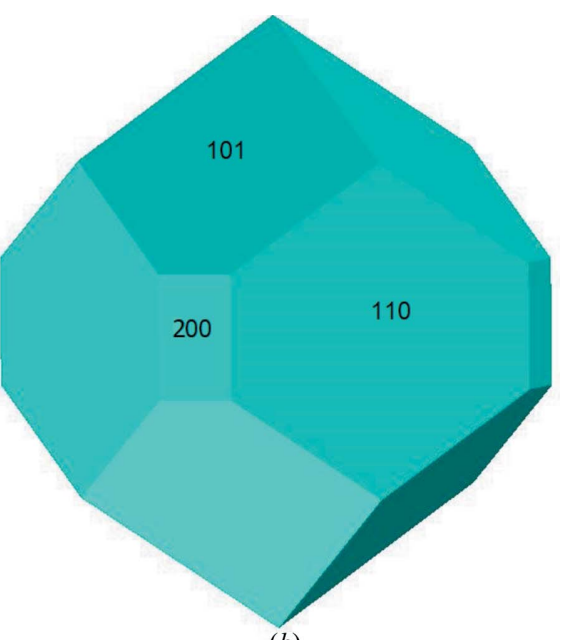

(b)
Figure 3

Growth morphology prediction by the AE method: $(a)$ unrelaxed and $(b)$ relaxed shape faces. 
Table 3

The calculated unrelaxed and relaxed $E_{\text {surf }}^{h k l}$ per unit formula, $R_{h k l}$ and $\mathrm{MI}_{h k l}$ of the different shape faces of the $\mathrm{GdVO}_{4}$ crystal in the SE method.

$R_{h k l}$ is $R_{(h k l)} / R_{(200)}$.

\begin{tabular}{llllllll}
\hline $\begin{array}{l}\text { Faces } \\
(h k l)\end{array}$ & $\begin{array}{l}d_{h k l} \\
(\mathrm{~A})\end{array}$ & $\begin{array}{l}\text { Unrelaxed } E_{\text {surf }}^{h k l} \\
\left(\mathrm{~J} \mathrm{~m}^{-2}\right)\end{array}$ & $R_{h k l}$ & $\mathrm{MI}_{h k l}$ & $\begin{array}{l}\text { Relaxed } E_{\text {surf }}^{h k l} \\
\left(\mathrm{~J} \mathrm{~m}^{-2}\right)\end{array}$ & $R_{h k l}$ & $\mathrm{MI}_{h k l}$ \\
\hline$(110)$ & 5.1233 & 0.1397 & 1.35 & 1.67 & 0.0942 & 1.08 & 1.05 \\
$(101)$ & 4.7860 & 0.2333 & 2.26 & 1.00 & 0.0985 & 1.13 & 1.00 \\
$(200)$ & 3.6227 & 0.1034 & 1.00 & 2.26 & 0.0872 & 1.00 & 1.13 \\
\hline
\end{tabular}

\& Jung, 2019), and theoretical prediction from Fig. 3(b) is confirmed by the experimentally observed crystal shape in Fig. 4(b) (Gavrilović et al., 2014).

It is surprising to find that an $S$ (stepped) face (in the Hartman-Perdok theory) can have a smaller attachment energy than an F (flat) face. Therefore, it could be possible if external conditions (high temperature and pressure like in the hydrothermal growth method) are taken into account. Under these circumstances, the (110) face changes its S character into

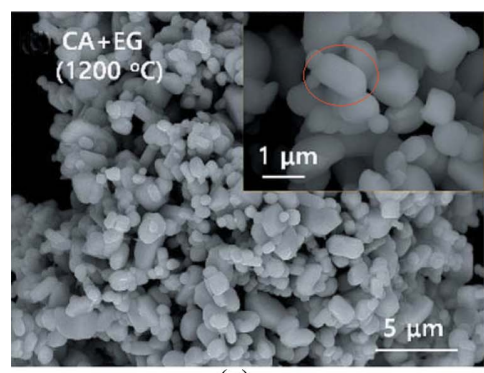

(a)

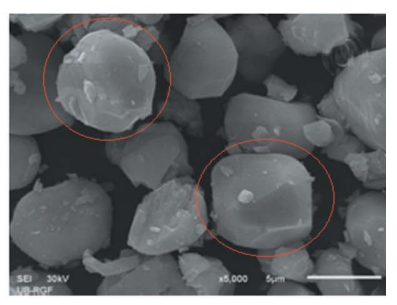

(b)
Figure 4

SEM images of $\mathrm{GdVO}_{4}$ doped with $\mathrm{Er}^{3+} / \mathrm{Yb}^{3+}$. (a) Reproduced with the permission of the Royal Society of Chemistry from Min \& Jung (2019); (b) reproduced with the permission of Elsevier from Gavrilović et al. (2014).

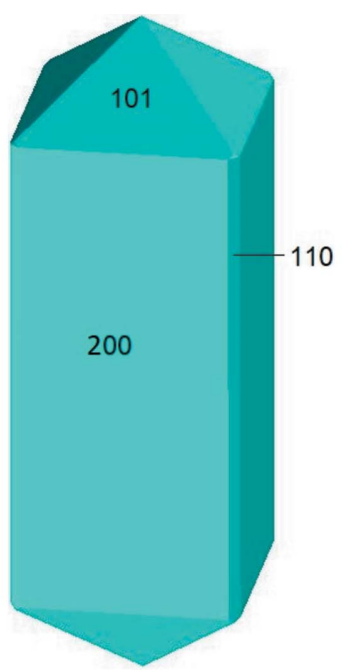

(a)

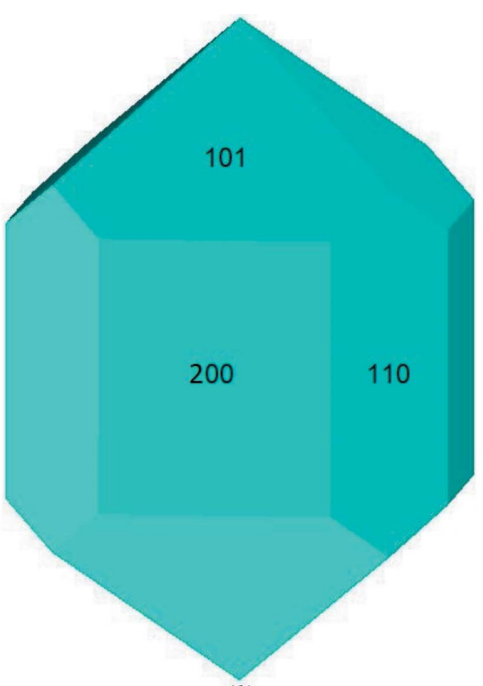

(b)
Figure 5

Equilibrium morphology prediction by the SE model: $(a)$ unrelaxed and (b) relaxed shape faces.
$\mathrm{F}$, and its contribution to the morphology prediction of the crystal becomes more important.

It can be concluded that the predicted morphologies compared favorably with the experimental morphologies. Compared with BFDH predicted morphology, the AE method shows the appearance of the (110) faces that become the most dominant in area, due to the fact that the energetic features of the crystal were taken into account. Despite the appearance of the (110) face by the AE method, the morphology predicted in the case of the relaxed structure has the same trend as the ranking provided by the BFDH method: $\mathrm{MI}_{101}>\mathrm{MI}_{200}$. As expected, the results gained from the $a b$ initio DFT by the AE model help us to improve the understanding of $\mathrm{GdVO}_{4}$ crystal growth mechanisms and to develop a more reliable and accurate morphology prediction than the BFDH model.

\subsection{Equilibrium morphology predictions by the SE model}

Based on equation (11), the calculated surface energies for (110), (101) and (200) faces, along with the corresponding values of $R_{h k l}$ and $\mathrm{MI}_{h k l}$ are listed in Table 3.

As can be seen from Table 3, the order of stability for the most morphologically important surfaces, suggested by $E_{\text {suf }}^{h k l}$ values, is the same for relaxed and unrelaxed structures: $\mathrm{MI}_{200}$ $>\mathrm{MI}_{110}>\mathrm{MI}_{101}$.

The theoretical equilibrium crystal shapes were obtained from these surface energy values and from the Wulff construction method [see Figs. 5(a), 5(b)]. The Wulff shape of the unrelaxed structure, Fig. 5(a), indicates a long bipyramid prism, while the Wulff shape of the relaxed structure, Fig. 5(b), indicates a short bipyramid prism. Both calculated shapes of $\mathrm{GdVO}_{4}$, using the SE method, match well with the experimental morphology of crystals from the work of CalderónVillajos et al. [2012, Fig. 6(a)] and Min \& Jung [2019, Fig. 6(b)].

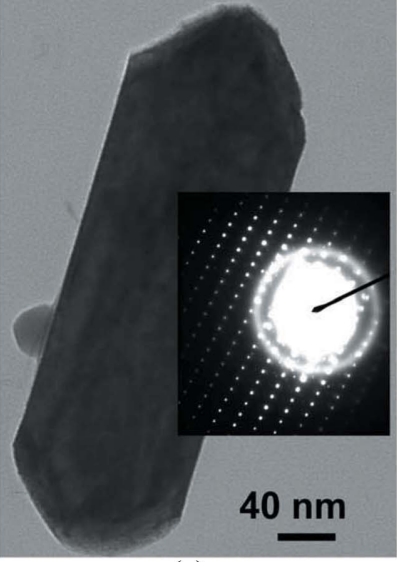

(a)

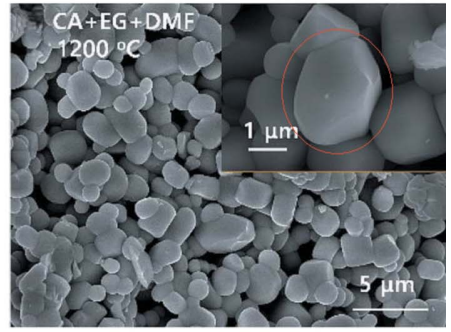

(b)
Figure 6

(a) TEM (transmission electron microscopy) image of synthetic crystal $\mathrm{GdVO}_{4}: \mathrm{Tm}^{3+}$ [reproduced with the permission of the Royal Society of Chemistry from Calderón-Villajos et al. (2012)]; (b) SEM microphotography of synthetic crystals $\mathrm{GdVO}_{4}: \mathrm{Er}^{3+} / \mathrm{Yb}^{3+}$ [reproduced with the permission of the Royal Society of Chemistry from Min \& Jung (2019)]. 
These morphologies show some similarity with that obtained with the AE method.

In light of these studies, based on first-principles DFT calculations, we have predicted several theoretical external morphologies of the $\mathrm{GdVO}_{4}$ crystal, connected with its internal structure, symmetry and energetics. The predicted morphologies depend on the method used and the application of relaxation.

All methods discussed above yielded morphologies which correspond, in a reasonable manner, to experimental crystals observed in nature or synthesized. Although the order of $\mathrm{MI}_{h k l}$ 's is different, the AE and SE methods based on the energetics of the system present the (110), (101) and (200) as the three most stable surfaces.

The BFDH method is an approximate method only, based on the geometry of the crystal unit cell and its symmetry. In the case of BFDH, only two faces (101) and (200) are retrieved as the most stable, which corresponds to the morphology of some experimentally observed crystals. Both the AE and SE methods take into account the energetics of the crystal. Finally, it is important to note that the main difference between the attachment energy method and surface energy method is that the first one gives information on the growth morphology and the second one on the equilibrium morphology of the crystal.

\section{Conclusions}

In this article, first-principles DFT calculations were performed in order to investigate the growth shape and equilibrium morphology of the $\mathrm{GdVO}_{4}$ crystal. The BFDH, $\mathrm{AE}$ and SE methods, in the frame of the $2 \mathrm{D}$ periodic slab model, were used. The slice energies, $E_{\text {coh }}, E_{\text {att }}^{h k l}$ and $E_{\text {surf }}^{h k l}$, of the main faces having (110), (101) and (200) orientation and their $d_{h k l}$ thicknesses were computed using CRYSTAL17 code. The morphologies predicted by each of the three methods were presented, discussed and compared with each other as well as with the experimentally observed morphologies.

Our results show a good consistency and have the ability to describe, in a reasonable manner, several experimentally observed $\mathrm{GdVO}_{4}$ crystal shapes.

The simplest, BFDH, theory predicts (101) and (200) faces to be of highest $\mathrm{MI}_{h k l}$, with the order $\mathrm{MI}_{101}>\mathrm{MI}_{200}$. The AE method gives a prediction of the crystal growth morphology with three $\mathrm{MI}_{h k l}$ faces (110), (101) and (200), for both unrelaxed and relaxed crystal geometries. The analysis of these three faces shows that the relaxation process of the crystal changes the order of $\mathrm{MI}_{h k l}$ for the faces (101) and (200). The SE method gives a prediction of the equilibrium crystal morphology, with $\mathrm{MI}_{h k l}$ faces (200), (110) and (101) and the unchanged order of $\mathrm{MI}_{h k l}$ for both unrelaxed and relaxed geometry.

Although the order of $\mathrm{MI}_{h k l}$ is different in $\mathrm{AE}$ and $\mathrm{SE}$ methods, they predict that the (110), (101) and (200) faces are the three most stable surface faces of the crystal. The agreement of the theoretical prediction from our study with experiment is quite satisfactory, in the sense that the broad features of the observed morphologies are reproduced. This justifies this new attempt to use first-principles DFT methodology in combination with $\mathrm{AE}$ and SE methods in the frame of predicting morphology of orthovanadate crystals.

\section{Acknowledgements}

We thank the Royal Society of Chemistry for permission to reproduce the Figs. 6(d) and 6(e) from Min \& Jung (2019) (an open access paper) and Fig. 7(a) from Calderón-Villajos et al. (2012). We also thank Elsevier for permission to reproduce Fig. 2(d) from Yan et al. (2013), and Fig. 3(d) from Gavrilovic et al. (2014).

\section{Funding information}

N. M. Avram thanks the West University of Timisoara for financial support under contract No. 61051/20/11.11.2019.

\section{References}

Aquilano, D., Otálora, F., Pastero, L. \& García-Ruiz, J. M. (2016). Prog. Cryst. Growth Charact. Mater. 62, 227-251.

Arrouvela, C. \& Eon, J.-G. (2019). Mater. Res. 22, e20171140.

Barbosa, M., Fabris, G. S. L., Ferrer, M. M., Azevedo, D. H. M. \& Sambrano, J. R. (2017). Mater. Res. 20, 920-925.

Becke, A. D. (1988). Phys. Rev. A, 38, 3098-3100.

Becke, A. D. (1993). J. Chem. Phys. 98, 1372-1377.

Belkina, I. O., Smolenko, N. P., Klochkov, V. K., Malukin, Y. V., Chistyakova, E. E., Karpenko, N. A. \& Karachentsev, Y. I. (2016). Fiziol. Zh. 62, 76-82.

Bennema, P. (1993). Handbook of Crystal Growth, Vol. 1a, ch. 7, edited by D. T. J. Hurle. Amsterdam: Elsevier Science Publishers.

Berkovitch-Yellin, Z. (1985). J. Am. Chem. Soc. 107, 8239-8253.

Bittarello, E., Bruno, M. \& Aquilano, D. (2018). Cryst. Growth Des. 18, 4084-4094.

Born, M. (1919). Verh. Dtsch. Phys. Ges. 21, 679-685.

Bravais, A. (1866). Etudes Cristallographiques. Paris: GauthierVillars.

Calderón-Villajos, R., Zaldo, C. \& Cascales, C. (2012). CrystEngComm, 14, 2756-2768.

Canepa, P., Hanson, R. M., Ugliengo, P. \& Alfredsson, M. (2011). J. Appl. Cryst. 44, 225-229.

Cante, S., Beecher, S. J. \& Mackenzie, J. I. (2018). Opt. Express, 26, 6478-6489.

Cho, S. (2013). j. Nanosci. Nanotech. 13, 7165-7168.

Chumha, N., Kittiwachana, S., Thongtem, T., Thongtem, S. \& Kaowphong, S. (2014). Ceram. Int. 40, 16337-16342.

Cong, H., Zhang, H., Sun, S., Yu, Y., Yu, W., Yu, H., Zhang, J., Wang, J. \& Boughton, R. I. (2010). J. Appl. Cryst. 43, 308-319.

Credendino, R., Busico, V., Causà, M., Barone, V., Budzelaar, P. H. M. \& Zicovich-Wilson, C. (2009). Phys. Chem. Chem. Phys. 11, 65256532.

Dandekar, P., Kuvadia, Z. B. \& Doherty, M. F. (2013). Annu. Rev. Mater. Res. 43, 13.1-13.28.

Desmarais, J. K., Erba, A. \& Dovesi, R. (2018). Theor. Chem. Acc. 137, 28-39.

Docherty, R., Clydesdale, G., Roberts, K. J. \& Bennema, P. (1991). J. Phys. D Appl. Phys. 24, 89-99.

Dong, H., Du, S.-R., Zheng, X.-Y., Lyu, G.-M., Sun, L.-D., Li, L.-D., Zhang, P.-Z., Zhang, C. \& Yan, C.-H. (2015). Chem. Rev. 115, 10725-10815.

Donnay, J. D. H. \& Harker, D. (1937). Am. Mineral. 22, 446-467.

Dovesi, R., Civalleri, B., Orlando, R., Roetti, C. \& Saunders, V. R. (2005). Reviews in Computational Chemistry, Vol. 21, edited by 
K. B. Lipkowitz, R. Larter \& T. R. Cundari. New Jersey: Wiley$\mathrm{VCH}$, John Wiley \& Sons.

Dovesi, R., Erba, A., Orlando, R., Zicovich-Wilson, C. M., Civalleri, B., Maschio, L., Rérat, M., Casassa, S., Baima, J., Salustro, S. \& Kirtman, B. (2018). WIREs Comput. Mol. Sci. 8, e1360.

Epelbaum, B. M., Shimamura, K., Inaba, K., Uda, S., Kochurikhin, V. V., Machida, H., Terada, Y. \& Fukuda, T. (1998). J. Cryst. Growth, 186, 607-611.

Friedel, G. (1907). Bull. Soc. Fr. Miner. 30, 326-455.

Friedel, G. (1911). Leçon de crystallographie. Paris: Hermann.

Gavezzotti, A. \& Filippini, G. (1997). The Molecular Solid State: Theoretical Aspects and Computer Modeling. New York: Wiley.

Gavrilović, T. V., Jovanović, D. J., Lojpur, V. M., Đorđević, V. \& Dramićanin, M. D. (2014). J. Solid State Chem. 217, 92-98.

Gavrilović, T. V., Jovanović, D. J., Smits, K. \& Dramićanin, M. D. (2016). Dyes Pigments, 126, 1-7.

Goel, S., Sinha, N., Yadav, H. \& Kumar, B. (2019). Phys. E: LowDimens. Syst. Nanostructures, 106, 291-297.

Gouveia, A. F., Ferrer, M. M., Sambrano, J. R., Andrés, J. \& Longo, E. (2016). Chem. Phys. Lett. 660, 87-92.

Haber, F. (1919). Verh. Dtsch. Phys. Ges. 21, 750-768.

Hartman, P. (1973). Editor. Crystal Growth: an Introduction, ch. 14. Amsterdam: North Holland.

Hartman, P. \& Bennema, P. (1980). J. Cryst. Growth, 49, 145-156.

Hartman, P. \& Perdok, W. G. (1955). Acta Cryst. 8, 521-524.

Heo, S. J., Batra, R., Ramprasad, R. \& Singh, P. (2018). J. Phys. Chem. $C$, 122, 28797-28804.

Huang, Z., Zhang, L., Feng, J., Cui, X. \& Pan, W. (2012). J. Alloys Compd. 538, 56-60.

Jenkins, H. D. B. (2005). J. Chem. Educ. 82, 950-952.

Jovanović, D. J., Chiappini, A., Zur, L., Gavrilović, T. V., Lam Tran, T. N., Chiasera, A., Lukowiak, A., Smits, K., Dramićanin, M. D. \& Ferrari, M. (2018). Opt. Mater. 76, 308-316.

Kevin, J. R., Docherty, R. \& Tamura, R. (2018). Editors. Engineering Crystallography: From Molecule to Crystal to Functional Form. Berlin: Springer.

Krasnikov, A., Tsiumra, V., Vasylechko, L., Zazubovich, S. \& Zhydachevskyy, Y. (2019). J. Lumin. 212, 52-60.

Lee, C. T., Yang, W. T. \& Parr, R. G. (1988). Phys. Rev. B, 37, 785-789.

Li, P., Zhao, X., Jia, C.-J., Sun, H., Li, Y., Sun, L., Cheng, X., Liu, L. \& Fan, W. (2012). Cryst. Growth Des. 12, 5042-5050.

Liang, X., Kuang, S. \& Li, Y. (2011). J. Mater. Res. 26, 1168-1173.

Liu, Y., Niu, S., Lai, W., Yu, T., Ma, Y., Gao, H., Zhao, F. \& Ge, Z. (2019). CrystEngComm, 21, 4910-4917.

Loiko, P. A., Yumashev, K. V., Matrosov, V. N. \& Kuleshov, N. V. (2013). Appl. Opt. 52, 698-705.

Macrae, C. F., Bruno, I. J., Chisholm, J. A., Edgington, P. R., McCabe, P., Pidcock, E., Rodriguez-Monge, L., Taylor, R., van de Streek, J. \& Wood, P. A. (2008). J. Appl. Cryst. 41, 466-470.

Mahapatra, S. \& Ramanan, A. (2005). J. Alloys Compd. 395, 149-153.

Massaro, F. R., Moret, M., Bruno, M., Rubbo, M. \& Aquilano, D. (2011). Cryst. Growth Des. 11, 4639-4646.

Mazierski, P., Sowik, J., Miodyńska, M., Trykowski, G., Mikołajczyk, A., Klimczuk, T., Lisowski, W., Nadolna, J. \& Zaleska-Medynska, A. (2019). Dalton Trans. 48, 1662-1671.
Min, B. H. \& Jung, K. Y. (2019). RSC Adv. 9, 20002-20008.

Nakayama, M., Hotta, S., Nakamura, T. \& Kasuga, T. (2013). J. Ceram. Soc. Japan, 121, 611-613.

Oliveira, M. C., Gracia, L., Nogueira, I. C., Carmo Gurgel, M. F., Mercury, J. M. R., Longo, E. \& Andrés, J. (2016). Ceram. Int. 42, 10913-10921.

Oshikiri, M., Ye, J. \& Boero, M. (2014a). J. Phys. Chem. C, 118, 83318341.

Oshikiri, M., Ye, J. \& Boero, M. (2014b). J. Phys. Chem. C, 118, 12845-12854.

Petrov, D. (2013). Croat. Chem. Acta, 87, 85-89.

Qiao, M., Wang, T., Song, H., Zhang, J., Liu, Y., Liu, P., Zhang, H. \& Wang, X. (2017). IEEE Photonics J. 9, 6101010.

Rabasovic, M. S., Krizan, J., Savic Sevic, S., Mitric, M., Rabasovic, M. D., Marinkovic, B. P. \& Sevic, D. (2018). J. Spectrosc. Article ID 3413864, 1-8.

Reshak, A. H. \& Azam, S. (2013). Int. J. Electrochem. Sci. 8, 1039610423.

Ribeiro, R. A. P., Lacerda, L. H. S., Longo, E., Andrés, J. \& de Lazaro, S. R. (2019). J. Magn. Magn. Mater. 475, 544-549.

Shanta Singh, N., Ningthoujam, R. S., Phaomei, G., Singh, S. D., Vinu, A. \& Vatsa, R. K. (2012). Dalton Trans. 41, 4404-4412.

Shimamura, K., Uda, S., Kochurikhin, V. V., Taniuchi, T. \& Fukuda, T. (1996). Jpn. J. Appl. Phys. 35, 1832-1835.

Singh, M. K., Banerjee, A. \& Gupta, P. K. (2012). J. Cryst. Growth, $\mathbf{3 4 3}, 77-85$.

Stirner, T., Scholz, D. \& Sun, J. (2018). Surf. Sci. 671, 11-16.

Szczeszak, A., Grzyb, T., Śniadecki, Z., Andrzejewska, N., Lis, S., Matczak, G., Nowaczyk, M., Jurga, S. \& Idzikowski, B. (2014). Inorg. Chem. 53, 12243-12252.

Tang, S., Huang, M., Wang, J., Yu, F., Shang, G. \& Wu, J. (2012). J. Alloys Compd. 513, 474-480.

Teo, R. D., Termini, J. \& Gray, H. B. (2016). J. Med. Chem. 59, 60126024.

Vasylechko, L., Tupys, A., Hreb, V., Tsiumra, V., Lutsiuk, I. \& Zhydachevskyy, Y. (2018). Inorganics, 6, 94-107.

Vilela Oliveira, D., Laun, J., Peintinger, M. F. \& Bredow, T. (2019). J. Comput. Chem. 40, 451-459.

Vosoughifar, M. (2017). J. Mater. Sci. Mater. Electron. 28, 61196124.

Wang, Z., Jiang, P. \& Dang, L. (2010). Proceedings of 4th International Conference on Bioinfomatics and Biomedical Engineering, 18-20 June 2010, Chengdu, pp. 1-4. Piscataway: IEEE.

Woensdregt, C. F. (1993). Faraday Discuss. 95, 97-107.

Wulff, G. (1901). Z. Kristallogr. 34, 449-530.

Yan, B. \& Gu, J.-F. (2009). J. Exp. Nanosci. 4, 301-311.

Yan, Y., Hojamberdiev, M., Xu, Y., Wang, J. \& Luan, Z. (2013). Mater. Chem. Phys. 139, 298-304.

Yu, H. H., Zhang, H. J. \& Wang, J. Y. (2013). Acta Phys. Pol. A, 124, 301-304.

Zhang, Y. (2014). Trop. J. Pharm. Res. 13, 829-834.

Zheng, F., Wang, W. \& Yang, P. (2011). Optoelectronics Adv. Mater. Rapid Commun. 5, 596-599.

Zhu, W., Jin, H. M., Wu, P. \& Liu, H. L. (2004). Phys. Rev. B, 70, 165419. 